\title{
Cerebellar cortical degeneration associated with feline leukemia virus infection and cerebellar lymphoma in a young cat
}

\author{
Alejandra Parra Pardoํㅜ Marcelo A. Gómez ${ }^{1 *}$ and Carlos M. González ${ }^{2}$ \\ ${ }^{1}$ Facultad de Ciencias Veterinarias, Universidad Austral de Chile, Valdivia, Chile \\ ${ }^{2}$ Escuela de Medicina Veterinaria, Facultad de Ciencias de la Vida, Universidad Andrés Bello, Santiago, Chile
}

\begin{abstract}
Background: Cerebellar cortical degeneration (CCD) is the premature death of cerebellar neurons of heterogeneous etiology that is uncommonly observed as a neurological complication of certain neoplasia.

Case Description: Here, we report an 8-month-old male domestic cat with altered consciousness, symmetric ataxia, hypermetric gait, vertical positional nystagmus, mydriasis, strabismus, intention tremor of the head, and increased patellar reflexes. Neuroanatomical diagnosis suggested a multifocal brain dysfunction (cerebellar and cerebral). The cat tested seropositive for feline leukemia virus. Cerebrospinal fluid analysis indicated mononuclear and neutrophilic pleocytosis. Contrast computed tomography imaging revealed multiple hypodense heterogeneous areas in both cerebral hemispheres, mild ventriculomegaly at the level of the caudal fossa, and a circular sharply marginated, homogeneously hyperdense mass occupying the right cerebellar hemisphere. Postmortem study indicated a $1.1 \times 1.3 \times 1.2 \mathrm{~cm}$ mass in the right cerebellar hemisphere close to the vermis. Histopathological analysis showed diffuse and severe Purkinje cell loss with a decrease in granular cell density and moderate gliosis compatible with CCD. Further, numerous neoplastic lymphoid cells were observed in the infiltrated mass, consistent with a diagnosis of central nervous system (CNS) lymphoma. Immunohistochemistry showed CD20 expression, indicative of a B-cell immunophenotype. In humans, CCD is reported as a rare paraneoplastic syndrome in patients with Hodgkin lymphoma. CNS lymphoma and/or Feline Leukemia Virus (FeLV) infection were both considered as a possible cause of CCD in this case.

Conclusion: This is the first described case of possible paraneoplastic cerebellar cortical degeneration associated with CNS lymphoma and/or FeLV infection in a domestic cat.

Keywords: Cat, Cerebellar degeneration, Feline leukemia virus, Lymphoma, Paraneoplastic syndrome.
\end{abstract}

\section{Introduction}

Cerebellar cortical degeneration (CCD) is defined as premature and irreversible degeneration of the cerebellum, caused by intrinsic or extrinsic factors leading to neuronal degenerative changes resulting in progressive signs of cerebellar dysfunction (Scott et al., 2018). The origin of CCD is heterogeneous, but in many cases, it is assumed to be associated with an autosomal recessive gene (Kent et al., 2000; Scott et al., 2018). Inflammatory-infectious, toxic, and immune-mediated etiologies should also be considered as underlying factors of CCD in domestic animals (Johnson et al., 1967; Percy et al., 1971; Scott et al., 2018). This neurological alteration is characterized by a substantial loss of Purkinje cells and the granular layer, and astrocytosis (Barone et al., 2002; Negrin et al., 2006; Biolatti et al., 2010). Cerebellar cortical degeneration has been described in humans as part of paraneoplastic neurological syndromes and also associated with viral infections (i.e, HIV) (Gungor et al., 2017; Pedroso et al., 2018).

Lymphoma is the second-most common intracranial tumor in cats (Mandara et al., 2016). Feline Leukemia
Virus (FeLV) infection has been associated with the presence of lymphomas in cats (Mandara et al., 2016). Cats with intracranial lymphoma have been reported to be positive for FeLV in $17.6 \%$ of the cases (Mandara et al., 2016). Histopathological criteria for the classification of feline lymphomas are based on cytomorphological, immunophenotypical, and mitotic indices (Weiss et al., 2010; Sato et al., 2014). In humans, based on histological and clinical characteristics, lymphomas are divided into Hodgkin or non-Hodgkin lymphomas (Ansell, 2015). Hodgkin lymphomas are composed of usually an abnormal type of B lymphocyte and associated with proliferative disorders (Ansell, 2015). Non-Hodgkin lymphomas are classified into B-cell and T-cell type, with 30 types of lymphoid cell neoplasms reported (Cheson et al., 2014). In humans, CCD is a rare paraneoplastic syndrome associated with Hodgkin lymphoma, small-cell lung cancer, breast cancer, and gynecological malignancies (Avramova et al., 2016; Yan et al., 2018). Paraneoplastic neurologic syndrome caused by FeLV-infected cats is associated with lymphoma and lymphocytic infiltration in the brain and spinal cord (Caemichael et al., 2002). Additionally, a polypeptide from some FeLV strains has shown to have a direct 
neurotoxic effect (Mitchell et al., 1997). Central nervous system (CNS) lymphoma or FeLV associated with CCD has not previously been reported in small animals.

The objective of this case report is to document the first case of CCD provably associated with feline leukemia virus infection and/or cerebellar lymphoma in a young domestic cat.

\section{Case Details}

An 8-month-old intact male, American shorthair, the indoor cat was presented to the Veterinary Hospital at Austral University of Chile (Valdivia, Chile) because of a 2-weeks history of incoordination and head tremor. Complete neurological examination found altered mental status (depressed), positional vertical nystagmus, bilateral mydriasis, unilateral ventral strabismus of the right eye, decreased menace response with a normal pupillary reflex, bilaterally hypermetric gait of all four limbs, intention tremor of the head, positive crossed extensor reflexes, and increased patellar reflex in both hind limbs. Neuroanatomical diagnosis indicated a central vestibular lesion with a multifocal brain type lesion (cerebellar and cerebral). Differential diagnosis included degenerative cerebellar and cerebral diseases such as lysosomal storage disease (i.e., gangliosidosis), brain malformation, hereditary cerebellar degeneration, cerebellar hypoplasia caused by infectious conditions (e.g., feline panleukopenia virus, FeLV, feline infectious peritonitis, toxoplasmosis, and feline spongiform encephalopathy disease), neoplastic conditions (e.g., lymphoma), toxicity (e.g., lead poisoning), congenital portosystemic shunt (i.e., hepatic encephalopathy), and CNS hypomyelination.

Additional tests included complete blood count, biochemical profile, FeLV/ Feline immunodeficiency virus (FIV) testing, cerebrospinal fluid analysis, and computed tomography (CT) of the head. Complete cell blood count showed no clinically significant abnormalities. Biochemical analysis results were within reference ranges. Cerebrospinal fluid indicated mononuclear and neutrophilic pleocytosis with normal total protein concentration $(<0.25 \mathrm{~g} / \mathrm{l})$. A commercial immunoassay was used to test blood samples for Feline leukemia virus antigen and FIV antibodies (SensPERT FeLV Ag/FIV Ab Test Kit, Vet All Laboratories). The cat tested positive for FeLV and negative for FIV. Contrast CT scanning of the head revealed multiple heterogeneous hypodense areas in both cerebral hemispheres, mild ventriculomegaly, and a circular sharply marginated homogeneously hyperdense mass occupying the right cerebellar hemisphere (Fig. 1A). The animal was treated with trimethoprim-sulfamethoxazole $(20 \mathrm{mg} / \mathrm{kg}$ every 12 hours) and prednisone (1 mg/kg every 24 hours) over 6 days due to the initial diagnosis of suspected systemic infection with neurologic signs or toxoplasmosis.

The cat responded poorly to therapy, and the owners requested humane euthanasia. A complete necropsy study was performed and postmortem macroscopic findings revealed a friable mass with regular edges of approximately $1.1 \times 1.3 \times 1.2 \mathrm{~cm}$ located in the right cerebellar hemisphere close to the vermis (Fig. 1B and 1C). On macroscopic examination, no abnormalities were observed in lung, heart, spleen, lymph node, large and small intestines. Cerebellar tissue sections were obtained for histopathological and immunohistochemical analyses. Cerebellar and cerebral tissue samples were fixed in 10\% formaldehyde, subjected to automatic tissue processing, and then embedded in paraffin. Thin sections of $5 \mu \mathrm{m}$ thickness were obtained and stained with hematoxylin and eosin. For immunohistochemical analysis of CNS lymphoma, antigenic recovery was performed using citrate buffer $\mathrm{pH}$. Endogenous peroxidase was blocked with a peroxide solution, while nonspecific reactivity was blocked by incubation with $10 \%$ normal goat serum (Thermo Fisher Scientific, São Paulo, Brazil). The primary antibodies used were an anti-CD3 antibody (Dako, Carpinteria, CA) at 1:50 dilution for 30 minutes, and anti-CD20 antibody (Thermo Fisher Scientific, USA) at 1:300 dilution for 30 minutes. Samples were subsequently incubated with mouse/rabbit Ig reagent (Vector Laboratories Inc., Burlingame, CA) for 30 minutes. Immunohistochemical analysis was performed using Histostain (Thermo Fisher Scientific, USA) with hematoxylin contrast staining.

Histopathological findings of both cerebellar hemispheres showed degenerative changes with neuronal death, white matter demyelination, and severe Purkinje cell loss (Fig. 2A and 2B). Additionally, decreased granule cell density and moderate gray matter gliosis were observed. In the cerebral tissue sections, changes included mild gliosis, white matter degeneration, and swollen axons.

Between the Purkinje cell and granular cell layers, degenerating neuronal cells were observed with eosinophilic, and occasionally, vacuolar cytoplasm, and regressive nuclear changes. Other histological findings included white matter spongiosis and axonal demyelination. Cerebellar nuclei showed isolated groups of eosinophilic cytoplasmic neurons, diffuse borders, and oncosis. These microscopic changes were associated with a morphological diagnosis of cerebellar degeneration.

Histological analysis of the cerebellar mass revealed infiltration of a uniform or monomorphic population of lymphocytic cells of moderate to severe atypia, compatible with lymphoma (Fig. 2C and 3A). The cells showed large, loose chromatin nuclei that presented with membrane irregularities, prominent nucleoli, and mitotic activity (average of 25 mitoses in 10 fields of $400 \times$ magnification). Additionally, multiple necrotic and apoptotic foci were observed inside the tissue mass. Immunostaining for CD20 expression identified a B-lymphocyte immunophenotype. More than 90\% of lymphoid cells were positive for CD20 (Fig. 3B), and occasionally, the presence of $\mathrm{T}$ lymphocytes was observed (CD3 positive). 


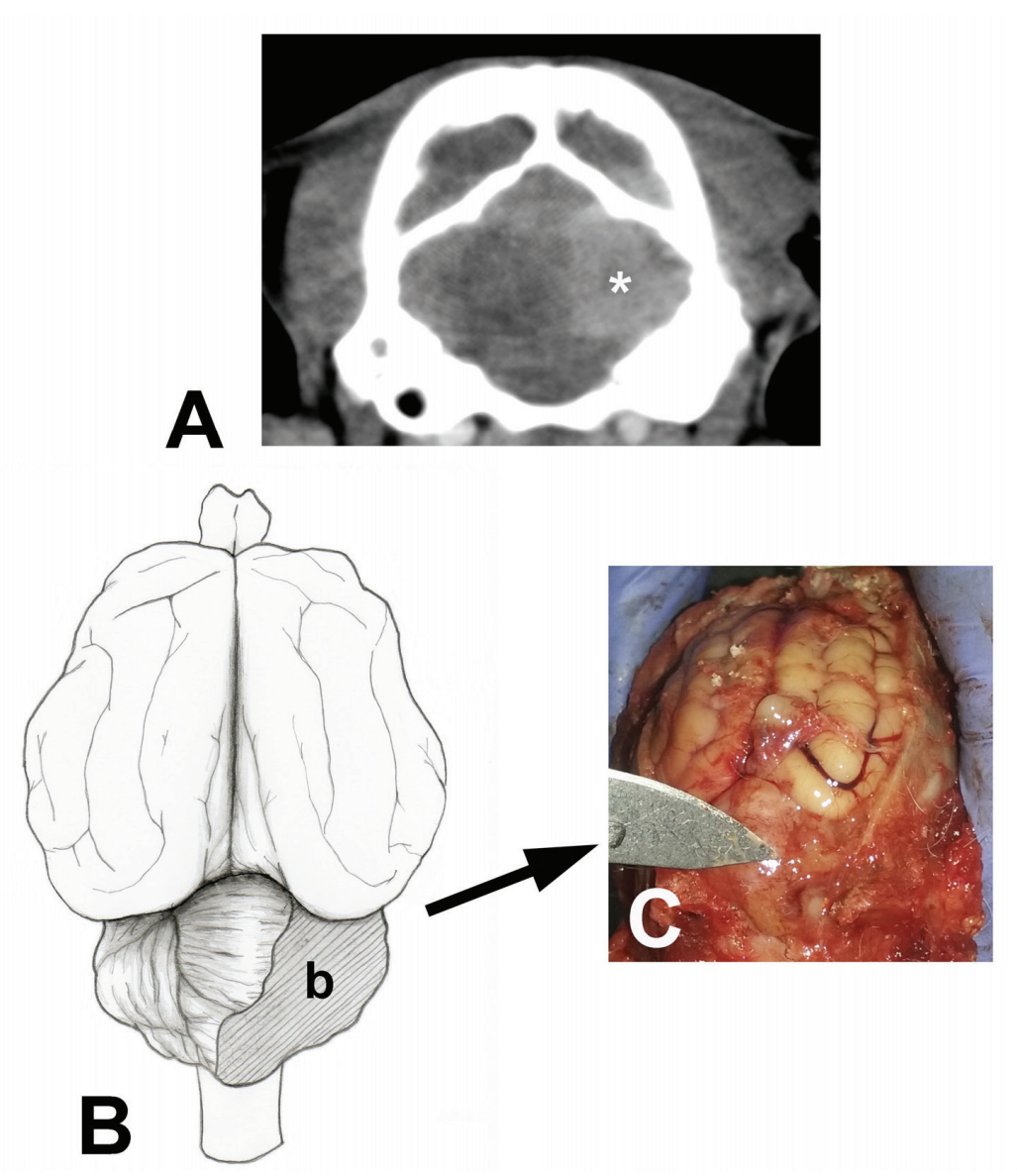

Fig. 1. (A). Contrast computed tomography of the head of an 8-month domestic cat with signs of dysmetria and head tremors. Note the homogenous sharply marginated hyperdense mass (white asterisk) in the right caudal cerebral fossa, which was consistent with cerebellar neoplasia. (B). Schematic representation of the location and extent of the neoplastic mass (b) in the cerebellum of a feline of 8 months of age. (C): Postmortem photographic image of the lesion. The neoplastic lesion included the vermis and right cerebellar hemisphere caudal to the primary fissure was later confirmed as lymphoma.

\section{Discussion}

The clinical signs described in this report are similar to previous cases of CCD including symmetric ataxia, hypermetric gait, spasticity, vertical nystagmus, absence of menace response, head tremors, opisthotonus, behavioral abnormalities, and depression (Shamir et al., 1999; Negrin et al., 2006; Biolatti et al., 2010). Differential diagnosis for CCD includes lysosomal storage disease, cerebellar hypoplasia (early presentation), neuroaxonal dystrophy, and feline spongiform encephalopathy disease (Shamir et al., 1999; Barone et al., 2002; Negrin et al., 2006; Biolatti et al., 2010; Scott et al., 2018). In the present case report, the neuropathological findings were confined to CNS. Microscopic changes in the cerebellum of the affected cat were consistent with previous cases of CCD and cerebellar primary lymphoma (Taniyama et al. 1994; Shamir et al., 1999; Barone et al., 2002; Negrin et al., 2006; Biolatti et al., 2010; Yoshino et al., 2017). However, no previous studies were found that simultaneously describe the presence of both pathological entities. The significance of small foci lesions detected on CT scan of this cat is unclear but probable due to inflammatory infiltration due to FeLV infection or vascular congestion due to lymphoma involvement. Similar microscopic changes have been observed in the spinal cord and brain stem parenchyma of FeLV-infected cats with associated-myelopathy (Caemichael et al., 2002).

The neuropathological pattern of CCD in cats is usually limited to Purkinje cells; however, retrograde reduction of the granular layer is also observed (granuloprival form of CCD) (Huska et al., 2013; Scott et al., 2018). Cerebellar cortical degeneration (CCD) is considered a neurodegenerative disorder of heterogeneous etiology (Scott et al., 2018). Using pedigree analysis, genomewide association studies, homozygosity mapping, and single nucleotide polymorphism, an inherited autosomal 


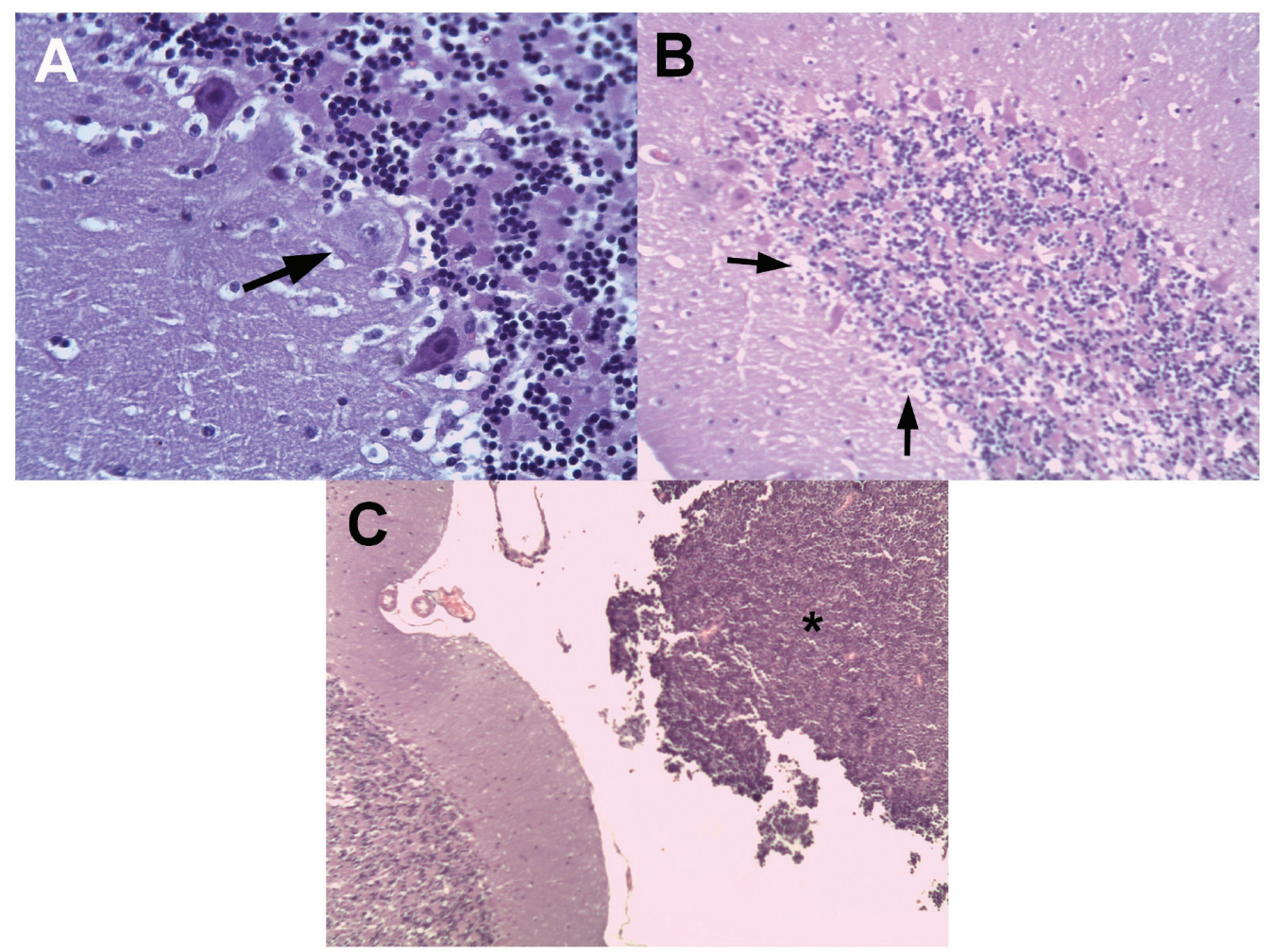

Fig. 2. (A). Degeneration of Purkinje cells with the presence of edematous cytoplasm and pyknotic nuclei (arrow). (B) Histological images (hematoxylin-eosin) of cerebellar folia of the left cerebellar hemisphere with characteristic cerebellar cortical degeneration showing decreased Purkinje cell number and empty baskets (small arrows). (C). Molecular and granular layers of a cerebellum folia, with a population of adjacent lymphoid neoplastic cells (black asterisk).

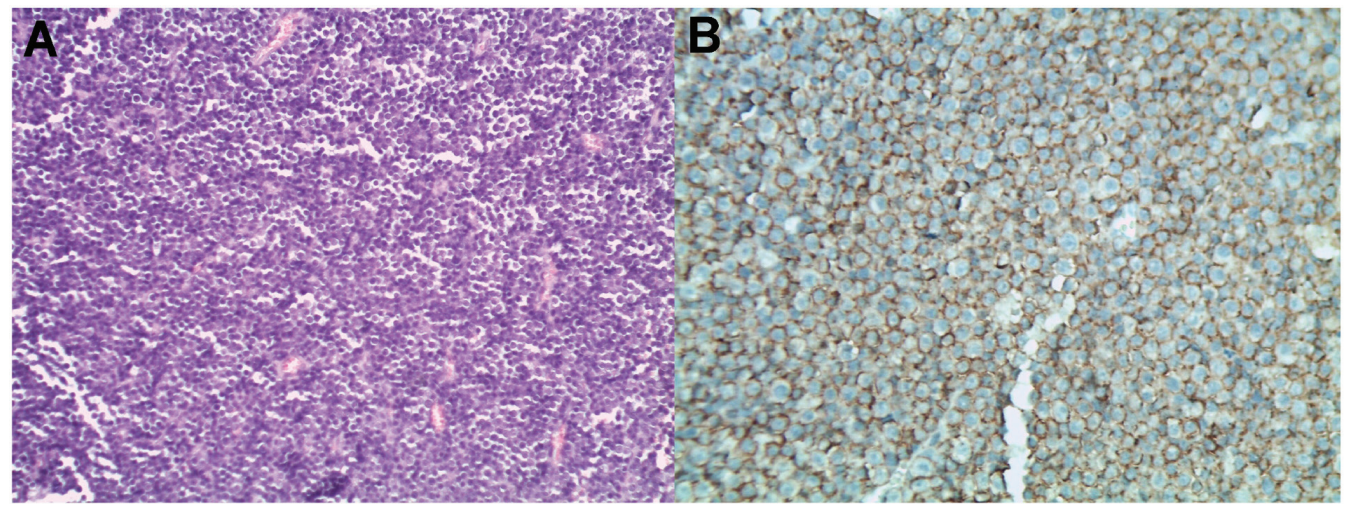

Fig. 3. Microscopic images of the cerebellar lymphoma (hematoxylin-eosin) in an 8-month-old domestic cat. (A) Monomorphic population of lymphocytic cells with severe atypia, which is compatible with cerebellar lymphoma. (B) Immunostaining for CD20 in a cerebellum sample from an 8-month-old domestic cat with progressive signs of cerebellar dysfunction. A generalized CD20-positive cell population $(>90 \%)$ compatible with B lymphocytes is observed.

recessive condition was associated with CCD in Finnish Hounds, Hungarian Vizsla dogs, and the Arabian horse (Scott et al., 2018). Alterations in expression of TOE1, $M U T Y H$, and $S N X 14$ genes have been proposed as molecular mechanisms (Brault et al., 2011; Scott et al.,
2018). Toxicity with alcohol, mercury, and drugs (such as phenytoin), and Solanum fastigiatum toxicosis have been reported to cause selective degeneration of Purkinje cells (Scott et al., 2018). Infectious agents should be also considered in the etiology of CCD in 
domestic animals (Scott et al., 2018). In dogs, canine herpes virus infection is suggested to produce CCD by reduction of granular and Purkinje cell populations and associated inflammatory mononuclear cells (Percy et al., 1971; Scott et al., 2018). Intrauterine or perinatal infection of feline panleukopenia virus reportedly causes cerebellar hypoplasia, with the presence of a decreased granular cell layer (Shamir et al., 1999). In humans, $\mathrm{CCD}$ is a rare paraneoplastic neurological syndrome associated with Hodgkin lymphoma as well as other tumor types (Avramova et al., 2016; Gungor et al., 2017). Polyclonal B-cell activation in human patients with Hodgkin lymphoma may be responsible for an autoimmune reaction in the cerebellum (Avramova et al., 2016).

Lymphomas are identified as the most common primary malignant tumor in domestic felines (Mandara et al., 2016). Approximately $30 \%$ of neoplasms in domestic felines are lymphomas (Troxel et al., 2003; Beatty, 2014). The etiology of lymphomas is described as multifactorial, although it is still not wellunderstood. Nonetheless, oncogenic viruses currently described in felines are constituted by retrovirus (FeLV provirus DNA), which are found in $13 \%-15 \%$ of cats with lymphomas (Beatty, 2014). Few reports have described feline lymphoma categories according to the World Health Organization classification (Sato et al., 2014). This classification is based on aspects of morphological, immunophenotypical, genetic, and clinical characteristics (Sato et al., 2014). Identification of B- or T-cell lineages is mandatory and usually determined by immunohistochemistry. CD20 is a suitable B-cell target antigen, which has an important function in activation, proliferation, and differentiation of B cells. Moreover, CD20 is a phosphoprotein that covers the membrane and represents a specific cellular antigen expressed in late B, pre B, and mature B cells. Other B-lymphoid cell markers include CD21, CD79a, BLA36, anti-IgM, anti-IgD, anti-IgG, anti-IgA, antikappa, and anti-lambda antibodies. For T lymphocytes, CD3, CD4, and CD8 are frequently used (Weiss et al., 2010; Beatty, 2014). Primary cerebellar B-cell lymphoma that displayed Hodgkin lymphoma-like tumor cells with FeLV infection has been reported in an adult domestic cat previously (Yoshino et al., 2017). CNS lymphoma is the most common neoplasm affecting the spinal cord and is the second-most common intracranial tumor in domestic cats (Troxel et al., 2003; Mandara et al., 2016). CNS lymphomas are distributed either peripherally or centrally, dividing into intracranial lymphomas, intraparenchymal lymphomas, angiotropic lymphomas, extra-axial lymphomas, lymphomatous choroiditis, lymphomatous meningitis, spinal cord lymphomas, or neurolymphomatosis (Troxel et al., 2003; Beatty, 2014; Mandara et al., 2016). Primary CNS lymphoma is a rare neoplasm in domestic felines, which often presents as a solitary, well-defined, and isolated intraparenchymal mass, or as a focally extensive infiltrate in the brain and/or spinal cord, which can be detected by CT, magnetic resonance imaging, or at necropsy examination (Troxel et al., 2003; Mandara et al., 2016; Yoshino et al., 2017).

Neurologic paraneoplastic syndromes are reported in felines with lymphomas, including peripheral paraneoplastic neuropathy, axonal loss, and demyelination (Cavana et al., 2009; Guil-Luna et al., 2013). As previously described (Pedroso et al., 2018), paraneoplastic CCD is a type of paraneoplastic neurological syndrome associated with Hodgkin lymphoma, and rarely with non-Hodgkin lymphoma in humans. Its pathophysiology involves neoplastic antigens and an immune response against tumor antigens, which results in leakage through the bloodbrain barrier, leading to secondary degeneration of cerebellar neurons, mainly affecting Purkinje cells (Avramova et al., 2016; Pedroso et al., 2018). Diagnosis involves western blotting for detection of paraneoplastic antibodies against Purkinje cells (anti$\operatorname{Tr}$ antibodies), and, frequently, these antibodies are associated with Hodgkin lymphoma and cerebellar degeneration (Avramova et al., 2016; Pedroso et al., 2018). In our report, it was not possible to detect anti-Tr antibodies since they are not available in felines so the paraneoplastic confirmation cannot be confirmed and remains speculative. Previously, a form of cerebellar granuloprival degeneration in Coton de Tuléar puppies was reported and autoimmune destruction of granular cells was observed resembling paraneoplastic CCD in humans (Tipold et al., 2000). Feline panleucopenia virus is able to express proteins and the replication cycle in post-mitotic Purkinje cells. In humans, cerebellar degeneration has been recently associated with HIVvirus infection probably by immune-mediated or direct neurotoxic virus effect. There are no reports of CCD associated with FeLV infection in felines or lymphoma in felines or other domestic animals. However, envelope polypetide protein (gp70) of the FeLV is known to cause dose-dependent neurotoxicity associated with alterations in intracellular calcium ion concentrations, neuronal survival, and neurite outgrowth. Unfortunately, FeLV p27 immunoreactive viral antigen was not performed in the cerebellum in order to confirm cerebellar neurons FeLV infection. In human medicine, it has been postulated that immune therapy (i.e., steroids, plasmapheresis, and monoclonal antibodies) can lead to a decrease in B cell number and reduction of CD20 antibodies in both serum and cerebrospinal fluid, which helps resolve paraneoplastic syndromes (Engelhard, 2016).

To the authors' knowledge, this is the first report of CCD in a young domestic cat with concomitant CNS intracranial lymphoma. It is possible that the present case of CCD may be etiologically associated with FeLV and/or CNS lymphoma. 


\section{Conclusions}

Cerebellar cortical degeneration is a neurodegenerative condition of rare presentation in domestic cats. In contrast, feline lymphoma is one of the most common CNS neoplasms, representing a great challenge in the daily clinic. Paraneoplastic cerebellar degeneration associated with lymphomas has not been reported and investigated in veterinary medicine. However, in humans, a variety of malignancies, such as lymphomas, are commonly associated with paraneoplastic CCD. Further research is needed to establish the possible causes of CCD attributed to paraneoplastic syndromes such as feline lymphoma.

\section{Acknowledgments}

The authors would like to thank Dr. Marcelo Mieres, School of Veterinary Medicine, Austral University of Chile, for his assistance in imaging diagnosis. We thank Rachel James, Ph.D., from Edanz Group (www.edanzediting.com/ac) for editing a draft of this manuscript. Part of this work was presented at the 31st Annual Symposium of the European Society of Veterinary Neurology (ESVN)-European College of Veterinary Neurology (ECVN), Copenhagen, Denmark, 2018.

\section{Conflict of interest}

The authors' declared that there is no conflict of interest. Author contributions

A.P.: Acquisition of data, drafting of the manuscript, assembled figures, critical revision of the manuscript, and approval of the article.

M.G.: Acquisition of data, critical revision of the manuscript, carried out the diagnostic assays, assembled figures, and approval of the article.

C.G.: Critical revision of the manuscript, carried out the diagnostic assays, and approval of the article.

\section{References}

Ansell, S.M. 2015. Hodgkin Lymphoma: Diagnosis and Treatment. Mayo Clin. Proc. 90, 1574-1583.

Avramova, B., Hristova, T., Yordanova, M., Vlahova, I., Muchinova, A., Bojinova, V. and Konstantinov, D. 2016. Cerebellar degeneration as a rare. Paraneoplastic syndrome in a child with Hodgkin Lymphoma. J. Pediatr. Hematol. Oncol. 6, 470472.

Barone, G., Foureman, P. and De Lahunta, A. 2002. Adult-onset cerebellar cortical abiotrophy and retinal degeneration in a domestic shorthair cat. J. Am. Anim. Hosp. Assoc. 38, 51-54.

Beatty, J. 2014. Viral causes of feline lymphoma: Retroviruses and beyond. Vet. J. 201, 174-180.

Biolatti, C., Gianella, P., Capucchio, M.T., Borrelli, A. and D'Angelo, A. 2010. Late onset and rapid progression of cerebellar abiotrophy in a domestic shorthair cat. J. Small Anim. Pract. 51, 123-126.

Brault, L.S., Cooper, C.A., Famula, T.R., Murray, J.D. and Penedo, M.C. 2011. Mapping of equine cerebellar abiotrophy to ECA2 and identification of a potential causative mutation affecting expression of MUTYH. Genomics 97, 121-129.

Caemichael, K.P., Bienzle, D. and McDonnell, J.J. 2002. Feline leukemia virus-associated myelopathy in cats. Vet. Pathol. 39, 536-545.

Cavana, P., Sammartano, F., Capucchio, M., Catalano, D., Valazza, A. and Farca, A.M. 2009. Peripheral neuropathy in a cat with renal lymphoma. J. Feline Med. Surg. 11, 869-872.

Cheson, B.D., Fisher, R.I., Barrington, S.F., Cavalli, F., Schwartz, L.H., Zucca, E. and Lister, T.A. 2014. Recommendations for initial evaluation, staging, and response assessment of Hodgkin and nonHodgkin lymphoma: the Lugano classification. J. Clin. Oncol. 32, 3059.

Engelhard, M. 2016. Anti-CD20 antibody treatment of non-Hodgkin lymphomas. Clin. Immunol. 172, 101-104.

Guil-Luna, S., Carrasco, L., Gómez-Laguna, J., Hilbe, M., Minguez, J.J., Kohler, K. and de las Mulas, J.M. 2013. Primary central nervous system T-cell lymphoma mimicking meningoencephalomyelitis in a cat. Can. Vet. J. 54, 602-605.

Gungor, S., Kilic, B., Arslan, M., Ozgen, U. and Dalmu, J. 2017. Hodgkin's lymphoma associated with paraneoplastic cerebellar degeneration in children: a case report and review of the literature. Childs Nerv. Syst. 33, 509-512.

Huska, J., Gaitero, L., Snyman, H., Foster, R.A., Pumarola, M. and Rodenas, S. 2013. Cerebellar granuloprival degeneration in an Australian Kelpie and a Labrador retriever dog. Can. Vet. J. 54, 55-60.

Johnson, R.H., Margolis, G. and Kilham, L. 1967. Identity of feline ataxia virus with feline panleucopenia virus. Nature 214, 175-177.

Kent, M., Glass, E. and Delahunta, A. 2000. Cerebellar cortical abiotrophy in a beagle. J. Small Anim. Pract. 41, 321-323.

Mandara, M.T., Motta, L. and Calo, P. 2016. Distribution of feline lymphoma in the central and peripheral nervous systems. Vet. J. 216, 109-116.

Mitchell, T.W., Rojko, J.L., Hartke, J.R., Mihajlov, A.R., Kasameyer, G.A., Gasper, P.W. and Whalen, L.R. 1997. FeLV envelope protein (gp70) variable region 5 causes alterations in calcium homeostasis and toxicity of neurons. J. Acquir. Immune Defic. Syndr. Hum. Retrovirol. 14, 307-320.

Negrin, A., Bernardini, M., Baumgartner, W. and Castagnaro, M. 2006. Late onset cerebellar degeneration in a middle-aged cat. J. Feline Med. Surg. 8, 424-429.

Pedroso, J.L., Vale, T.C., Gama, M.T.D., Ribas, G., Kristochik, J.C.G., Germiniani, F.M.B., Fink, M.C.D.D.S., Oliveira, A.C.P., Teive, H.A.G. and Barsottini, O.G. 2018. Cerebellar degeneration and progressive ataxia associated with HIV-virus infection. Parkinsonism Relat. Disord. 54, 95-98. 
Percy, D.H., Carmichael, L.E., Albert, D.M., King, J.M. and Jonas, A.M. 1971. Lesions in puppies surviving infection with canine herpesvirus. Vet. Pathol. 8, 37-53.

Sato, H., Fujino, Y., Chino, J., Takahashi, M., GotoKoshino, Y., Uchida, K. and Tsujimoto, H. 2014. Prognostic analyses on anatomical and morphological classification of feline lymphoma. J. Vet. Med. Sci. 76, 807-811.

Scott, E.Y., Woolard, K.D., Finno, C.J. and Murray, J.D. 2018. Cerebellar abiotrophy across domestic species. Cerebellum 17, 372-379.

Shamir, M., Perl, S. and Sharon, L. 1999. Late onset of cerebellar abiotrophy in a Siamese cat. J. Small Anim. Pract. 40, 343-345.

Taniyama, H., Takayanagi, S., Izumisawa, Y., Kotani, T., Kaji, Y., Okada, H. and Matsukawa, K. 1994. Cerebellar cortical atrophy in a Kitten. Vet. Pathol. 31, 710-713.

Tipold, A., Fatzer, R., Jaggy, A., Moore, P. and Vandevelde, M. 2000. Presumed immune mediated cerebellar granuloprival degeneration in the Coton de Tuléar breed. J. Neuroimmunol. 110, 130-133.

Troxel, M.T., Vite, C.H., Van Winkle, T.J., Newton, A.L., Dayrell-Hart, B., Kapatkin, A.S., Shofer, F.S. and Steinberg, S.A. 2003. Feline intracranial neoplasia: retrospective review of 160 cases (19852001). J. Vet. Intern. Med. 17, 850-859.

Weiss, A., Klopfleisch, R. and Gruber, A. 2010. Prevalence of feline leukaemia provirus DNA in feline lymphomas. J. Feline Med. Surg. 12, 929935.

Yan, L., Dong, X., Xu, H., Huang, J., Wang, W., Huang, L., Wan, Q. and Gong, J. 2018. Paraneoplastic cerebellar degeneration associated with breast cancer: A case report and review of the literature. Mol. Clin. Oncol. 9, 163-167.

Yoshino, Y., Chambers, J.K., Nakamori, T., GotoKoshino, Y. Nishigaki, K., Tsujimoto, H., Matsuki, N., Nakayama, H., Uchida, K. 2017. Primary cerebellar lymphoma with Hodgkin lymphomalike morphology in a cat. J. Vet. Diagn. Invest. 29, 707-710. 